[4] C. C. Lee, "Fuzzy logic in control systems: Fuzzy logic controller-Part II," IEEE Trans. Syst., Man, Cybern., vol. 20, pp. 419-435, 1990.

[5] J. B. Kiska, M. M. Gupta, and P. N. Nikkiforuk, "Energetics stability of fuzzy dynamic systems," IEEE Trans. Syst., Man, Cybern., vol. SMC-15, pp. 783-792, 1985.

[6] J. Maiers and Y. S. Sherif, "Applications of fuzzy set theory," IEEE Trans. Syst., Man, Cybern., vol. 15, pp. 175-189, 1988.

[7] Perry, "Case study: From lab to market quickly," IEEE Spectrum, pp. $64-65$, Oct. 1990.

[8] K. S. Ray and D. D. Majumber, "Application of circle criteria for stability analysis of linear SISO and MIMO systems associated with fuzzy logic controller," IEEE Trans. Syst., Man, Cybern., vol. SMC-14, pp. 345-349, 1984.

[9] K. Self, "Designing with fuzzy logic," IEEE Spectrum, pp. 42-44, Nov. 1990.

[10] T. Takegi and M. Sujeno, "Fuzzy identification of systems and its applications to modeling and control," IEEE Trans. Syst., Man, Cybern., vol. SMC-15, pp. 116-132, 1985.

[11] K. C. Tang and R. J. Mulholland, "Comparing fuzzy logic with classical controller designs," IEEE Trans. Syst., Man, Cybern., vol. SMC-17, pp 1085-1087, 1987.

[12] L. A. Zadeh, "Outline of a new approach to the analysis of complex systems and decision processes," IEEE Trans. Syst., Man, Cybern., vol. SMC-3, pp. 28-44, 1973.

[13] L. A. Zadeh, K. S. Fu, K. Taneka, and M. Shimura, Fuzzy Sets and Their Applications. New York: Academic, 1975.

\section{Determining the Collision-Free Joint Space Graph for Two Cooperating Robot Manipulators}

Qing Xue, Anthony A. Maciejewski, and P. C.-Y. Sheu

\begin{abstract}
The problem of path planning for two planar robot manipulators that cooperate in carrying a rectangular object from an initial position and orientation to a destination position and orientation in a 2-D environment is investigated. In this approach, the two robot arms, the carried object and the straight line connecting the two robot bases together are modeled as a 6 -link closed chain. The problem of path planning for the 6-link closed chain is solved by two major algorithms: the collision-free feasible configuration finding algorithm and the collisionfree path finding algorithm. The former maps the free space in the Cartesian world space to the robot's joint space in which all the collisionfree feasible configurations (CFFC's) for the 6-link closed chain are found. The latter builds a connection graph of the CFFC's and the transitions between any two groups of CFFC's at adjacent joint intervals. Finally, a graph search method is employed to find a collision-free path for each joint of the robot manipulators. The proposed algorithms can deal with cluttered environments and is guaranteed to find a solution if one exists.
\end{abstract}

\section{INTRODUCTION}

There is an increasing need for robots to replace human beings at dangerous or tedious jobs in manufacturing, in nuclear reactors, under the sea, or in outer space. In these applications, the coordination of multiple cooperating robots plays an important role. For instance, in

Manuscript received January 20, 1990; revised September 19, 1991, and July 2,1992 .

Q. Xue is with the School of Electrical Engineering, Purdue University at Indianapolis, 723 W. Michigan Street, Indianapolis, IN 46202-1285.

A. A. Maciejewski is with the Department of Electrical Engineering, Purdue University, West Lafayette, IN 47907-5132.

P. C.-Y. Sheu is with the Department of Electrical and Computer Engineering, Rutgers University, Piscataway, NJ 08855-0909.

IEEE Log Number 9205802. the case where an object that needs to be carried by a single robot is too large or too heavy, the coordination of two robot arms must be provided. Generally speaking, motions executed by two cooperating arms can be classified into two groups: loosely coordinated motions and tightly coordinated motions [1]. The major difference between them is that in loosely coordinated motions, two robot arms execute two independent working sequences for two unrelated tasks, but share a common working space. On the other hand, in tightly coordinated motions, two robot arms execute two related working sequences for a common task. This paper addresses the problem of collision-free path planning for two tightly coordinated planar robot arms in a known environment.

The problem of collision-free path planning for robots has been studied for many years. However, most research on the path planning problem has focused on a single robot. Among them, the configuration space method [2], [3] and the method for solving the piano movers' problem [4]-[6] have received the most attention. These methods have been further developed for many different applications in [7]-[13], particularly in view of reducing the computational complexity.

When robotics research is extended from single arm operation to coordinated multiple arm operation, many problems unique to robot coordination emerge. These problems, include, but are not limited to, kinematic coordination [14], dynamic coordination [15], load distribution among robots [16], and minimum-time trajectory planning [17]. Many researchers have examined these problems in the last few years. Compared to the large number of new approaches and results in the fields of kinematics and dynamic control, optimal load distribution, and minimum trajectory planning, little progress has been achieved in the field of collision-free path planning for tightly coordinated robots

Collision-free path planning for coordinated robot coordination is a relatively new research topic. An overview of existing approaches is as follows: The problem of collision avoidance among two (or multiple) loosely coordinated robots was investigated by Freund and Hoyer [18], [19]. An approach to solve the problem of planning a collision-free path for two tightly cooperating robot arms was given by Fortune, Wilfong and Yap [20]. Another approach to the same problem, was proposed by Zapata, Fournier and Dauchez [21]. In the former approach, the robot manipulators were restricted to be Stanford robot arms (spherical robot arms). In the latter approach, objects were approximated as a combination of several spheres.

In this paper, we will describe an alternate approach to the path planning problem for two planar robot manipulators with three revolute joints that cooperate in carrying a rectangular object from an initial position and orientation to a destination position and orientation in a 2-D environment. This approach can deal with cluttered environments and polygonal obstacles. In addition, the proposed approach converges, that is, a collision-free path from the initial position and orientation of the carried object to the final position and orientation of the carried object will be found if it exists. The central concept in this approach is to first locate the free space in joint space and then compute a set of paths for the two robot manipulators by using a graph search method in the joint free space. The novelty of the approach presented here is that it combines both approximate and exact components of the cell decomposition technique. This is done by determining a mapping that allows one to reduce the four coupled degrees of freedom in the system into a single parameter and then to use the approximate cell-decomposition on the 


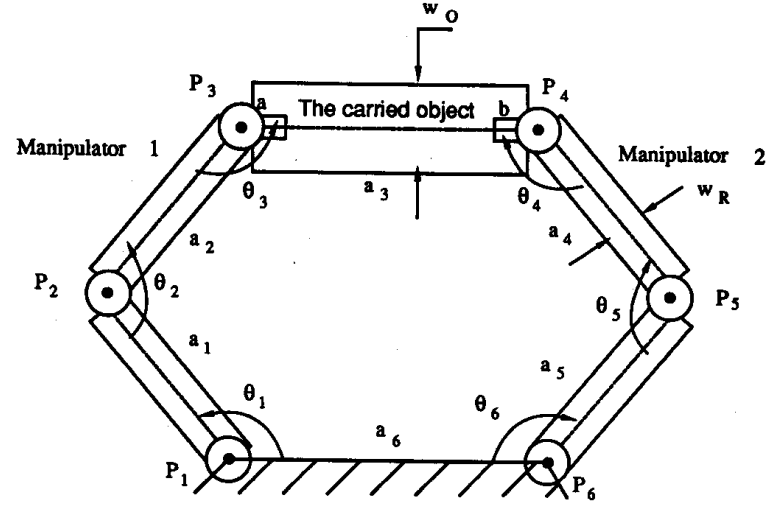

Fig. 1. Two planar three-degree-of-freedom manipulators that grasp an object and form a 6-link closed chain.

remaining two dimensions. This has the advantage of providing an exact answer to the dimension of the problem in which a mathematical analysis can not be avoided as well as a lower dimensional component in which approximate cell decomposition can be efficiently applied. For simplicity, the dynamics of the moving object is not considered. Furthermore, it is assumed that all information about the environment is available (in contrast to work on collision avoidance in uncertain environments [22]) so that path planning is performed off-line.

\section{Problem formulation}

In our approach for finding the paths of two closely cooperating planar manipulators that carry a rectangular object in a 2-D environment, it is assumed that each robot manipulator consists of three revolute joints and the end-effectors of the two robot manipulators grasp the rectangular object at points $a$ and $b$, which are the intersections of the boundary and the centerline of the rectangular object (see Fig. 1). For convenience, point $a$ is chosen as the reference position for the carried object with the orientation defined as the angle between the centerline of the rectangular object and the $x$-axis of the world coordinate system. Furthermore, it is assumed that there are $q$ stationary obstacles that are represented by polygons.

In the following discussion, the links of the robot manipulators, the carried rectangular object and the straight line connecting the two robots bases together are modeled as a 6-link closed chain. In the closed chain, the upper and the lower links of one manipulator, the carried object, the lower and the upper links of the other manipulator and the line segment connecting two robot bases are denoted as link $a_{1}$ to $a_{6}$ respectively (see Fig. 1).

The length of each link $a_{i}$ in the 6-link closed chain is denoted as $l_{i}$. It is assumed that all links of the manipulators have the same width $w_{R}$ and the carried rectangular object has width $w_{O}$. Furthermore, the centerlines of links $a_{i}$ and $a_{i+1}$ are assumed to be connected by a revolute joint that is located at point $P_{i+1}$ and the centerlines of link $a_{1}$ and link $a_{6}$ are assumed to be connected by the revolute joint that is located at point $P_{1}$. The angle of the joint located at $P_{i}$ is denoted as $\theta_{i}$. The direction of $\theta_{i}$ is defined in Fig. 1, where the joint angles of one manipulator increase in the counter clockwise direction, while the joint angles of the other manipulator increase in the clockwise direction.

Our problem is to plan a path for each joint of the 6-link closed chain so that the carried object can be moved from a given starting position and orientation to a given final position and orientation without colliding with obstacles. To find the collision-free path for the 6-link closed chain, the following constraints must be satisfied:
1) The Closed-Chain Constraint: The adjacent links should always be connected to each other.

2) The Link Collision-Free Constraints.

a) If two links are adjacent, one link is not allowed to pass through the other link and no link can intersect the carried rectangular object. In other words, $\theta_{i}$ must be in the range $\left[0^{\circ}, 360^{\circ}\right]$ for $i=1,2,5$, or 6 . The ranges for joint angles $\theta_{3}$ and $\theta_{4}$ are based on the size of the object as well as on the physical limits of the actuator and may be anywhere in the range $\left[0^{\circ}, 360^{\circ}\right]$. For the sake of illustration, the range $\left[90^{\circ}, 270^{\circ}\right]$ will be used throughout the remainder of this work.

b) The nonadjacent links in the 6-link closed chain are not allowed to intersect one another.

3) The Obstacle Collision-Free Constraint: The 6-link closed chain must not intersect any obstacles.

If these constraints are satisfied, then there is no collision between the robot links, between the robot links and the carried object, and between the 6-link closed chain and any obstacles in the environment.

For convenience, the joint angles in the 6-link closed chain are represented as a 6-tuple $\left(\theta_{1}, \theta_{2}, \theta_{3}, \theta_{4}, \theta_{5}, \theta_{6}\right)$. An instance of the 6-tuple that satisfies the closed-chain constraint is defined to be a configuration of the 6-link closed chain. A configuration that satisfies the link collision-free constraints is defined to be a feasible configuration. Furthermore, a feasible configuration that does not collide with any obstacles is defined to be a collision-free feasible configuration, which is denoted as CFFC. It is assumed that the configurations of the 6-link closed chain at the given initial and final positions and orientations of the carried object (link $a_{3}$ ) are collision-free and feasible.

Our goal is to find a sequence of collision-free feasible configurations that connects the initial CFFC and the final CFFC. This task can be achieved in two steps. In the first step, a collision-free feasible configuration finding algorithm is employed to find all the CFFC's for the closed chain at each quantized interval of two of the six joint angles. The second step is composed of three parts. First, a collision free path finding algorithm is employed to find the transitions between two groups of CFFC's at each pair of adjacent joint intervals. Second, a connection graph whose vertices are groups of CFFC's and whose edges are transitions between groups of CFFC's at adjacent joint intervals is built. Finally, a collision-free path is computed in the connection graph.

\section{Finding Collision-Free Feasible Configurations}

It is well-known from mechanics that a planar four bar linkage has only one independent degree of freedom [23]. Therefore in order to move a 6-link closed chain as described in Section II from one configuration to another configuration, the positions of at least three consecutive links in the closed chain need to be changed. For convenience, any link of the closed chain in which the position of one or both ends can be changed is called a changeable link. It can be seen that all links, except $a_{6}$, in the 6-link closed chain are changeable links. Subsequently, each set of three consecutive changeable links $a_{i}, a_{i+1} a_{i+2}$, where $1 \leq i \leq 3$, in a 6 -link closed chain is defined to be a basic changeable unit $B C U\left(a_{i}, a_{i+1}, a_{i+2}\right)$. Therefore, there are three basic changeable units $B C U\left(a_{1}, a_{2}, a_{3}\right), B C U\left(a_{2}, a_{3}, a_{4}\right)$ and $B C U\left(a_{3}, a_{4}, a_{5}\right)$ in a 6 -link closed chain. Since there is one degree of freedom of motion in a BCU, there are three degrees of freedom of motion in a 6-link closed chain. In other words, there are only three independent joint variables among 6 joints with the other three being dependent variables. 
Our strategy to find all the CFFC's for a 6-link closed chain is to find all the CFFC's generated by a $\mathrm{BCU}$ for each quantized motion of the other two changeable links that are not in the $\mathrm{BCU}$. The reason for not using an analytic approach is that there is no simple mapping from the three dependent joint variables to the independent variables under consideration of all the constraints discussed in Section II. For illustration, in the following discussion $B C U\left(a_{2}, a_{3}, a_{4}\right)$ is arbitrarily chosen and the motion of links $a_{1}$ and $a_{5}$ of the closed chain is quantized. The procedure would be identical if either of the other two BCU's is selected. In the course of finding CFFC's, the constraints given in Section II are satisfied one by one in the following subsections.

\section{A. Finding All the Configurations for $B C U\left(a_{2}, a_{3}, a_{4}\right)$}

As defined earlier, a configuration of a closed chain is an instance of the 6-tuple $\left(\theta_{1}, \theta_{2}, \theta_{3}, \theta_{4}, \theta_{5}, \theta_{6}\right)$ which satisfies the closed-chain constraint. Since $B C U\left(a_{2}, a_{3}, a_{4}\right)$ corresponds to three changeable links $a_{2}, a_{3}$, and $a_{4}$ that are adjacent and connected in a 6-link closed chain, if the other two changeable links $a_{1}$ and $a_{5}$ which are not in $B C U\left(a_{2}, a_{3}, a_{4}\right)$ are fixed, the position changes of the three changeable links in $B C U\left(a_{2}, a_{3}, a_{4}\right)$ will generate a set of configurations that satisfy the closed-chain constraint. Conșequently, our strategy to find all the configurations for the closed chain is to find all the configurations for $B C U\left(a_{2}, a_{3}, a_{4}\right)$ at each quantized position of the other two changęable links $a_{1}$ and $a_{5}$. Since the closed-chain constraint requires that each pair of adjacent links be connected to each other, it can be observed in Fig. 1 that a necessary condition to satisfy the closed-chain constraint in $B C U\left(a_{2}, a_{3}, a_{4}\right)$ is that $\left|P_{2} P_{5}\right| \leq l_{2}+l_{3}+l_{4}$, where $\left|P_{2} P_{5}\right|$ represents the distance between points $P_{2}$ and $P_{5}$. For convenience, it is assumed that this constraint holds in the following discussion.

Since $B C U\left(a_{2}, a_{3}, a_{4}\right)$ has a single degree of freedom, it can be represented by a single parameter. It can be observed that in $B C U\left(a_{2}, a_{3}, a_{4}\right)$, if the position of one link (called the active link) is changed, the positions of the other two links (called the passive links) must be changed accordingly. Since the positions of links $a_{2}$ and $a_{4}$ can be uniquely described by joint angles $\theta_{2}$ and $\theta_{5}$ respectively, it is convenient to choose one of them as the active link and choose the corresponding joint angle to represent $B C U\left(a_{2}, a_{3}, a_{4}\right)$. In the following discussion, it is assumed that $a_{4}$ is chosen as the active link and $B C U\left(a_{2}, a_{3}, a_{4}\right)$ is described by the parameter $\theta_{5}$. Hence, the task of finding all the configurations can be solved by finding the motion range of $\theta_{5}$, denoted as $M R\left(\theta_{5}\right)$, in which the closed-chain constraint can be satisfied. In order to find $M R\left(\theta_{5}\right)$, the following definitions are introduced.

Definition 1: If one end of the centerline of a changeable link $a_{i}$ is fixed at a point $P$, then all the possible positions for the other end of the centerline of $a_{i}$ form a circle with radius $l_{i}$ and center $P$. This circle is called the link position circle of $a_{i}$ and is denoted as $L P C_{a_{i}}(P)$ or simply $L P C_{a_{i}}$. $\square$

Definition 2: The circles that have a common center $P_{2}$ and radii $\left(l_{2}+l_{3}\right)$ and $\left|l_{2}-l_{3}\right|$ are defined as circle I and circle II, respectively. $\square$

Based on the triangle inequality concept, it can be shown [24] that the motion range $M R\left(\theta_{5}\right)$ in which the closed-chain constraint is satisfied should be a set of regions on the link position circle $L P C_{a_{4}}\left(P_{5}\right)$, which lies within circle I, but outside circle II. As an example, if circle I intersects $L P C_{a_{4}}$ at $h_{1}$ and $h_{2}$, but circle II does not intersect $L P C_{a_{4}}$, the motion range $M R\left(\theta_{5}\right)$ is equal to the range $\left[<P_{6} P_{5} h_{2},<P_{6} P_{5} h_{1}\right]$ on $L P C_{a_{4}}$ (see Fig. 2).

For a given position of the active link $a_{4}$ or a given $\theta_{5}$ in $M R\left(\theta_{5}\right)$, the possible positions of $P_{3}$ (which connects the passive links $a_{2}$ and $\left.a_{3}\right)$ should be on both $L P C_{a_{2}}\left(P_{2}\right)$ and $L P C_{a_{3}}\left(P_{4}\right)$ (see Fig. 3).

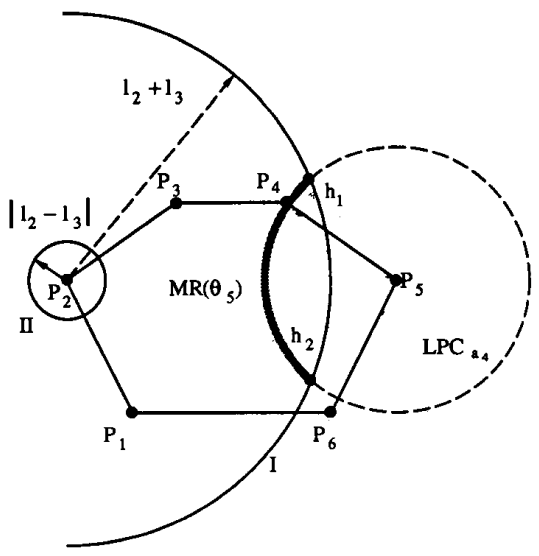

Fig. 2. An illustration of the motion range of $\theta_{5}$ (denoted $\operatorname{MR}\left(\theta_{5}\right)$ ), which satisfies the closed-chain constraint.

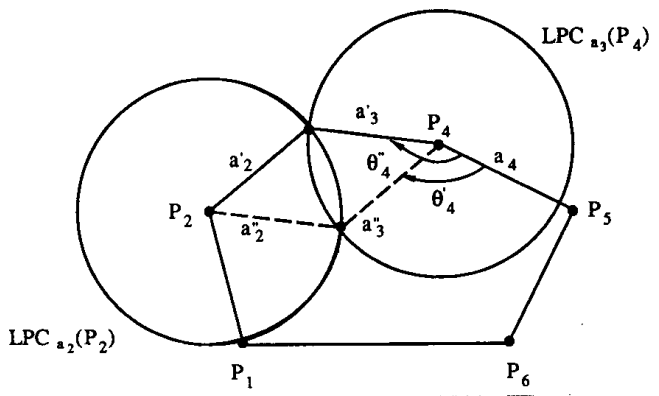

Fig. 3. An illustration of the two symmetric configurations that result from a single position of $a_{4}$. The configuration shown in the solid line is called a large configuration and the one in the dashed line is called a small configuration.

Hence, the possible positions for $P_{3}$ should be at the intersections of $L P C_{a_{2}}$ and $L P C_{a_{3}}$. Since $l_{2}+l_{3}+l_{4}<\left|P_{2} P_{5}\right|, L P C_{a_{2}}$ and $L P C_{a_{3}}$ have two intersections so that two possible positions of $P_{3}$ can be found. In other words, a given position of $a_{4}$ or a given $\theta_{5}$ in $M R\left(\theta_{5}\right)$ can correspond to two possible positions for the passive links $a_{2}$ and $a_{3}$ and therefore two configurations can be obtained. Thus all of the configurations of $B C U\left(a_{2}, a_{3}, a_{4}\right)$ are included in this motion range.

\section{B. Finding All the Feasible Configurations for $B C U\left(a_{2}, a_{3}, a_{4}\right)$}

In this section, we shall find a set of subregions of the motion range $M R\left(\theta_{5}\right)$ in which the link collision-free constraints are satisfied. These subregions of $M R\left(\theta_{5}\right)$ are called the feasible regions and are denoted by $F R\left(\theta_{5}\right)$. Once $F R\left(\theta_{5}\right)$ is found, all the feasible configurations of $B C U\left(a_{2}, a_{3}, a_{4}\right)$ can be determined by restricting the motion of $\theta_{5}$ to $F R\left(\theta_{5}\right)$.

The link-collision-free constraints resulting from the requirement that the links not collide with one another will be considered in two categories based on whether the links are or are not adjacent. The motivation for this distinction lies in the observation that collision between adjacent links can be prevented by limiting the joint ranges of the associated joints while collisions between nonadjacent links must consider more geometric constraints.

1) Finding Subregions of $M R\left(\theta_{5}\right)$, which Satisfy the Link Collision-Free Constraint for Adjacent Links: It is easy to see that one can guarantee that the adjacent links of $B C U\left(a_{2}, a_{3}, a_{4}\right)$ are collision 


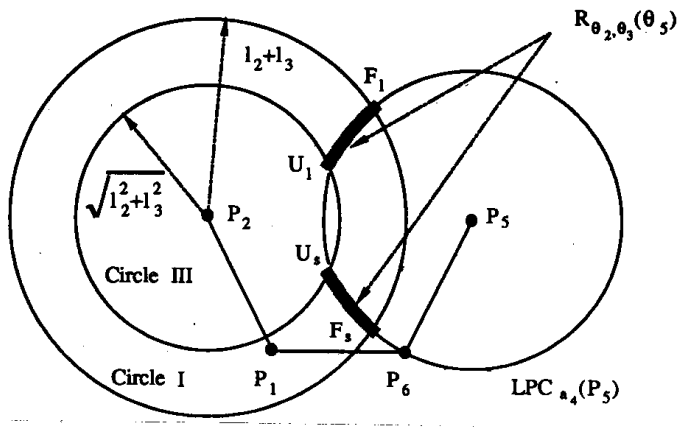

Fig. 4. A graphic interpretation of the mapping from the joint limit constraints on $\theta_{2}$ and $\theta_{3}$ onto ranges of $\theta_{5}$, denoted by $R_{\theta_{2}, \theta_{3}}\left(\theta_{5}\right)$.

free by restricting the range of $\theta_{2}$ and $\theta_{5}$ to be between 0 and 360 degrees and $\theta_{3}$ and $\theta_{4}$ to be between 90 and 270 degrees. Since $\theta_{5}$ is chosen as a parameter to represent $B C U\left(a_{2}, a_{3}, a_{4}\right)$, our approach is to map these constraints onto the motion range of $\theta_{5}$. This mapping is performed in the following two steps:

1) The constraint on the range of $\theta_{2}$ and the constraint on the range of $\theta_{3}$ are mapped onto ranges of $\theta_{5}$.

2) The constraint on $\theta_{4}$ is first mapped to ranges of $\theta_{2}$ in a procedure similar to step (1) and then the resulting ranges of $\theta_{2}$ are mapped onto ranges of $\theta_{5}$.

The resulting set of valid motion ranges for $\theta_{5}$ are then further decomposed in order to distinguish regions which posses single solution from those which have dual symmetric solutions. The need for this decomposition arises from the fact that the inverse kinematic mapping for revolute manipulators is multiple valued.

Mapping the valid joint ranges of $\theta_{3}$ and $\theta_{2}$ onto the ranges of $\theta_{5}$ : As mentioned previously, the valid range of $\theta_{3}$ is between 90 and 270 degrees and the valid range of $\theta_{2}$ is between 0 and 360 degrees. Since $\theta_{5}$ was chosen as the parameter to represent $B C U\left(a_{2}, a_{3}, a_{4}\right)$, the valid ranges of $\theta_{3}$ and $\theta_{2}$ should be mapped to ranges of $\theta_{5}$. These ranges of $\theta_{5}$ are denoted as $R_{\theta_{2}, \theta_{3}}\left(\theta_{5}\right)$. In other words, if $\theta_{5} \epsilon$ $R_{\theta_{2}, \theta_{3}}\left(\theta_{5}\right)$, the corresponding configurations have $\theta_{3} \in\left[90^{\circ}, 270^{\circ}\right]$ and $\theta_{2} \in\left[0^{\circ}, 360^{\circ}\right]$.

To find $R_{\theta_{2}, \theta_{3}}\left(\theta_{5}\right)$, we consider links $a_{2}$ and $a_{3}$ as a two link manipulator in which the base is located at $P_{2}$ and the end-effector is located at $P_{4}$. It can be observed in Fig. 4 that to guarantee $\theta_{3} \in\left[90^{\circ}, 270^{\circ}\right]$ and $\theta_{2} \in\left[0^{\circ}, 360^{\circ}\right]$, the workspace of this two link manipulator is bounded by a ring. The outer boundary circle of the ring is circle I with center $P_{2}$ and radius $l_{2}+l_{3}$. The interior boundary circle of the ring is denoted as circle III, which has center $P_{2}$ and radius $\sqrt{l_{2}^{2}+l_{3}^{2}}$ (see Fig. 4). By the closed-chain constraint, $a_{3}$ and $a_{4}$ must be connected. Consequently, $R_{\theta_{2}, \theta_{3}}\left(\theta_{5}\right)$ will be a set of regions on the link position circle $L P C_{a_{4}}$, which lie within the outer boundary circle I, but outside the interior boundary circle III. Based on the relative positions between circles I, III and $L P C_{a_{4}}$, $R_{\theta_{2}, \theta_{3}}\left(\theta_{5}\right)$ will fall into one of following three cases.

Case 1: If $L P C_{a_{4}}$ is inside circle III, then $R_{\theta_{2}, \theta_{3}}\left(\theta_{5}\right)=\Phi$.

Case 2: If $L P C_{a_{4}}$ is outside circle III, but intersects circle I at points $F_{l}$ and $F_{s}$, then $R_{\theta_{2}, \theta_{3}}\left(\theta_{5}\right)=\left[<P_{6} P_{5} F_{l},<P_{6} P_{5} F_{s}\right]$.

Case 3: If circle III intersects $L P C_{a_{4}}$ at points $U_{l}$ and $U_{s}$ and circle I intersects $L P C_{a_{4}}$ at points $F_{l}$ and $F_{s}$, then $R_{\theta_{2}, \theta_{3}}\left(\theta_{5}\right)=$ $\left[<P_{6} P_{5} F_{s},<P_{6} P_{5} U_{s}\right] \cup\left[<P_{6} P_{5} U_{l},<P_{6} P_{5} F_{l}\right]$.

As an example, case 3 is shown in Fig. 4. It can be observed from the previous three cases that $R_{\theta_{2}, \theta_{3}}\left(\theta_{5}\right)$ may contain two disjoint ranges of $\theta_{5}$ values, a single valid range, or no valid range. Since $I$ and III have center $P_{2}$ and $L P C_{a_{4}}$ has center $P_{5}$, a possible single valid

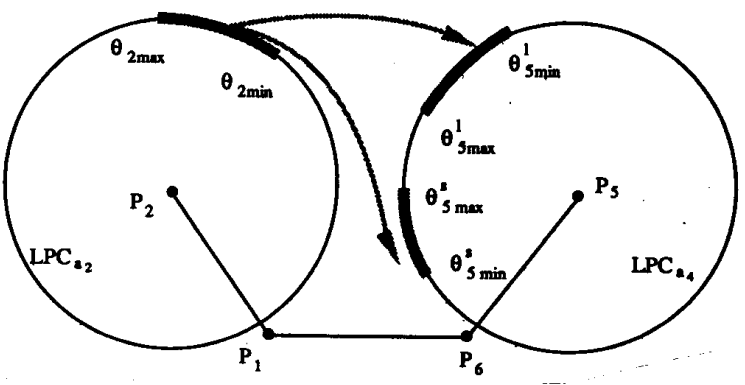

Fig. 5. One range of $\theta_{2}$ can be mapped to two ranges of $\theta_{5}$.

range of $\theta_{5}$ or two possible valid disjoint ranges will be symmetric with respect to $\overline{P_{2} P_{5}}$, where $\overline{P_{2} P_{5}}$ represents the straight line between points $P_{2}$ and $P_{5}$.

Mapping the valid range of $\theta_{4}$ onto ranges of $\theta_{5}$ : Since it is not easy to directly map the valid range of $\theta_{4}$ onto ranges of $\theta_{5}$, the valid range of $\theta_{4}$ is first mapped onto ranges of $\theta_{2}$, which are denoted $R_{\theta_{4}}\left(\theta_{2}\right)$, then $R_{\theta_{4}}\left(\theta_{2}\right)$ is mapped to ranges of $\theta_{5}$, which are denoted $R_{\theta_{4}}\left(\theta_{5}\right)$. Thus for each $\theta_{5} \in R_{\theta_{4}}\left(\theta_{5}\right)$, at least one corresponding configuration has $\theta_{4} \in\left[90^{\circ}, 270^{\circ}\right]$.

To obtain $R_{\theta_{4}}\left(\theta_{2}\right)$, a procedure that is similar to finding $R_{\theta_{2}, \theta_{3}}\left(\theta_{5}\right)$ can be used. After finding $R_{\theta_{4}}\left(\theta_{2}\right)$, the closed-chain constraint upon $R_{\theta_{4}}\left(\theta_{2}\right)$ is checked by an approach similar to that given in Section III-A. Subsequently, the valid range of $R_{\theta_{4}}\left(\theta_{2}\right)$, which satisfies the closed-chain constraint can be mapped to a set of regions of $\theta_{5}$, i.e., $R_{\theta_{4}}\left(\theta_{5}\right)$. To find this $R_{\theta_{4}}\left(\theta_{5}\right)$, the following definition needs to be introduced.

Definition 3: In $B C U\left(a_{2}, a_{3}, a_{4}\right)$, for any given $\theta_{2}$, there are two possible configurations. In order to distinguish between these two configurations, it can be noted that the $\theta_{3}$ in one configuration is larger than that in the other configuration. The term small configuration with respect to $\theta_{2}$ or simply small configuration will be used to refer to the configuration that has the smaller value of $\theta_{3}$. Similarly, the term large configuration with respect to $\theta_{2}$ or simply large configuration will be used to refer to the configuration that has the larger value of $\theta_{3}$. The analogous definition can be made for a given value of $\theta_{5}$ based on the value of $\theta_{4}$. These two configurations are illustrated in Fig. 3. $\square$

Since one $\theta_{2}$ corresponds to two configurations or two values of $\theta_{5}$, a range of $\theta_{2}$ can be mapped to two ranges of $\theta_{5}$. In order to find this mapping, it is assumed that there is a valid range of $R_{\theta_{4}}\left(\theta_{2}\right)$, i.e., $R_{\theta_{4}}\left(\theta_{2}\right)=\left[\theta_{2 \min }, \theta_{2 \max }\right]$. To map $\left[\dot{\theta}_{2 \min }, \theta_{2 \max }\right]$ to the ranges of $\theta_{5}$, the two boundaries $\theta_{2}$ min and $\theta_{2} \max$ are mapped first. Since both $\theta_{2 \min }$ and $\theta_{2 \max }$ correspond to two values of $\theta_{5}$ (one for small configuration and one for large configuration). For convenience, it is assumed that $\theta_{2 \mathrm{~min}}$ is mapped to $\theta_{5 \mathrm{~min}}^{s}$ (for the small configuration) and $\theta_{5 \mathrm{~min}}^{l}$ (for the large configuration). Similarly, $\theta_{2 \max }$ is assumed to be mapped to $\dot{\theta}_{5 \max }^{s}$ (for the small configuration) and $\theta_{5 \max }^{l}$ (for the large configuration). Thus two ranges that are the range between $\theta_{5 \min }^{l}$ and $\theta_{5 \max }^{l}$ for the large configurations and the range between $\theta_{5 \min }^{8}$ and $\theta_{5 \max }^{8}$ for the small configurations can be obtained (see Fig. 5). Unfortunately, it can be shown that there exist cases in which the range $\left[\theta_{5 \min }^{l}, \theta_{5 \max }^{l}\right]$ or the range $\left[\theta_{5 \min }^{s}, \theta_{5 \max }^{s}\right]$ is not the complete mapping of $\left[\theta_{2 \min }, \theta_{2 \max }\right]$. As an example, consider the case shown in Fig. 6. When $\theta_{2}$ decreases from $\theta_{2 \max }$ to $\theta_{2 \min }, \theta_{5}$ increases from $\theta_{5 \max }^{l}$ to some point $A$, and then starts to decrease from $A$ to $\theta_{5 \mathrm{~min}}^{l}$. To explain this phenomenon, a property of $B C U\left(a_{2}, a_{3}, a_{4}\right)$ is introduced.

Property 1: In $B C U\left(a_{2}, a_{3}, a_{4}\right)$, if $l_{2}+l_{3}<\left|P_{2} P_{5}\right|+l_{4}$, then $a_{2}$ and $a_{3}$ can become aligned. When $a_{2}$ and $a_{3}$ are aligned, $\theta_{5}$ 


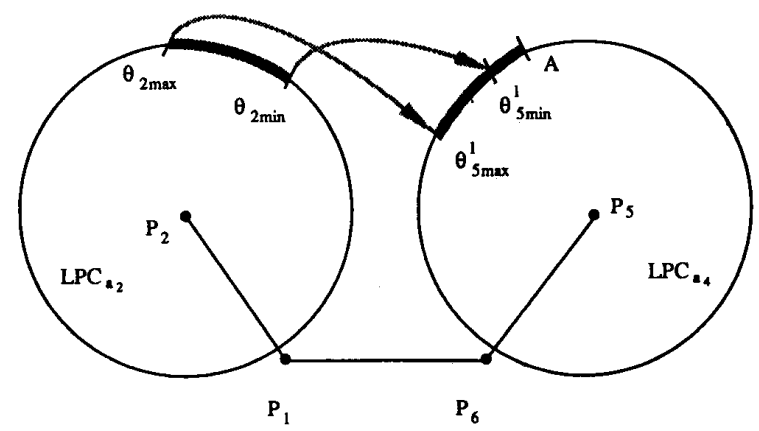

Fig. 6. An illustration of the case where the extremal values of the range of $\theta_{2}$ are not mapped to extremal value of the range of $\theta_{5}$.

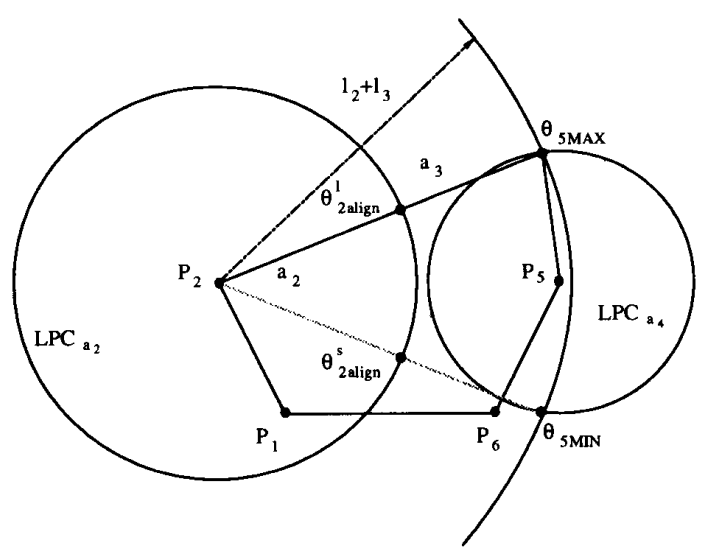

Fig. 7. If $\left|l_{2}+l_{3}\right|<\left|P_{2} P_{5}\right|+l_{4}$, then not all values of $\theta_{5}$ can satisfy the closed-chain constraint. The maximum and minimum allowable values of $\theta_{5}$ denoted $\theta_{5} \max$ and $\theta_{5}$ min, occur when links $a_{2}$ and $a_{3}$ are aligned. The $\theta_{2}$. The $\theta_{2}$ values in these cases are denoted $\theta_{2}^{l}$ align and $\theta_{2}^{s}$ align, respectively.

will reach its maximum value $\theta_{5 \max }$ or its minimum value $\theta_{5} \min$. The value of $\theta_{2}$ is denoted as $\theta_{2 \mathrm{align}}^{l}$ if $\theta_{5}=\theta_{5 \max }$ or $\theta_{2 \mathrm{align}}^{\mathrm{s}}$ if $\theta_{5}=\theta_{5 \text { min }}$.

This property can be explained by Fig. 7. It can be shown in Fig. 7 that if the condition $l_{2}+l_{3}<\left|P_{2} P_{5}\right|+l_{4}$ holds, the circle with center $P_{2}$ and radius $l_{2}+l_{3}$ can intersect $L P C_{a_{4}}$ so that $a_{2}$ and $a_{3}$ can become aligned. When $a_{2}$ and $a_{3}$ are aligned, the distance between $P_{2}$ and $P_{4}$ reaches its maximum. Consequently, $\theta_{5}$ cannot become any larger or smaller.

Based on Property 1 , if $\theta_{2 \text { align }}^{l}$ or $\theta_{2 \text { align }}^{s}$ is not inside the valid range of $\theta_{2}$, when $\theta_{2}$ monotonically decreases from one boundary to another boundary, corresponding $\theta_{5}$ will monotonically increases or decreases from one boundary to another boundary. For illustration, in the case of the large configurations (the case for the small configurations can be discussed in a similar way), it can be shown in Fig. 8 that when $\theta_{2 \min }, \theta_{2 \max }>\theta_{2 \mathrm{align}}^{l}$, if $\theta_{2}$ decreases from $\theta_{2 \text { max }}$ to $\theta_{2 \mathrm{~min}}$, the distance between $P_{2}$ and $P_{4}$ increases so that $\theta_{5}$ increases. Thus $\left[\theta_{2 \min }, \theta_{2 \max }\right]$ is mapped to $\left[\theta_{5 \text { max }}^{l}, \theta_{5 \text { min }}^{l}\right]$. In Fig. 9 , since $\theta_{2 \min }, \theta_{2 \max }<\theta_{2 \mathrm{align}}^{l}$, if $\theta_{2}$ decreases from $\theta_{2 \text { max }}$ to $\theta_{2 \mathrm{~min}}$, the distance between $P_{2}$ and $P_{4}$ also decreases so that $\theta_{5}$ decreases. Thus $\left[\theta_{2 \min }, \theta_{2 \max }\right]$ is mapped to $\left[\theta_{5 \min }^{l}, \theta_{5 \max }^{l}\right]$. However, if $\theta_{2 \text { align }}^{l}$ or $\theta_{2 \text { align }}^{s}$ is inside the range of $\theta_{2}$, the previous two cases should be combined. For instance, since the case shown in Fig. 6 has $\theta_{2 \min } \leq \theta_{2 \mathrm{align}}^{l} \leq \theta_{2 \max }$, when $\theta_{2}$ decreases from $\theta_{2 \max }$ to $\theta_{2 \mathrm{align}}^{l}$,
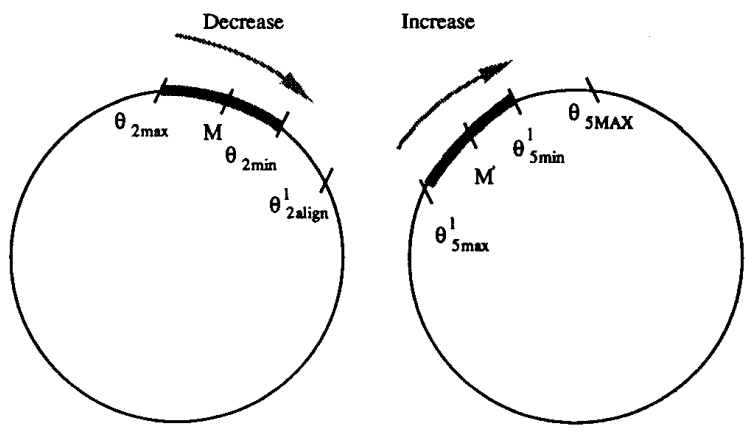

(a)

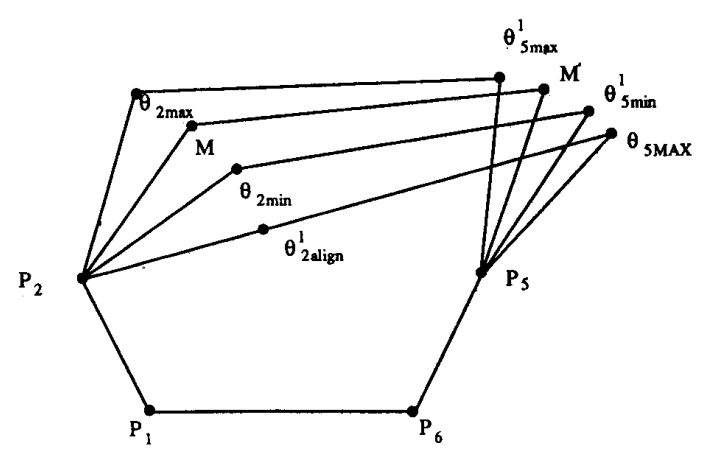

(b)

Fig. 8. If all values in the range $\left[\theta_{2 \min } \theta_{2 \max }\right]$ are larger than $\theta_{2 \mathrm{align}}^{1}$ then $\left[\theta_{2 \min } \theta_{2 \max }\right]$ is mapped to $\left[\theta_{5}^{l} \max \theta_{5}^{l} \min \right]$

$\theta_{5}$ increases from $\theta_{5 \text { max }}^{I}$ to $A$ that is equal to $\theta_{5 \text { max }}$ and $a_{2}$ and $a_{3}$ become aligned. If $\theta_{2}$ continues to decrease from $\theta_{2 \mathrm{align}}^{l}$ to $\theta_{2 \mathrm{~min}}, \theta_{5}$ starts to decrease from $\theta_{5 \max }$ to $\theta_{5 \min }^{l}$. Consequently, [ $\left.\theta_{2 \min }, \theta_{2 \max }\right]$ is mapped to $\left[\min \left(\theta_{5 \min }^{l}, \theta_{5 \max }^{l}\right), \theta_{5 \max }\right]$. The previous discussion can be summarized in the algorithm given previously.

Algorithm 1: To map the range $\left[\theta_{2 \mathrm{~min}}, \theta_{2 \mathrm{max}}\right]$ to the ranges of $\theta_{5}$,

1) map the boundaries of the range $\left[\theta_{2} \min , \theta_{2}\right.$ max $]$ to the ranges of $\theta_{5}$, i.e., map $\theta_{2 \text { min }}$ and $\theta_{2}$ max to $\theta_{5 \min }^{l}$ and $\theta_{5 \max }^{l}$ for the large configurations respectively, and $\theta_{5 \min }^{s}$ and $\theta_{5 \max }^{s}$ for the small configurations respectively.

2) map $\theta_{2}$ between the boundaries of the range $\left[\theta_{2 \min }, \theta_{2 \max }\right]$ to the ranges of $\theta_{5}$.

a) for the large configurations, the following three cases hold:

- if $\theta_{2 \max }, \theta_{2 \min }>\theta_{2 \text { align }}^{l}$, then $\left[\theta_{2 \min }, \theta_{2 \max }\right]$ is mapped to $\left[\theta_{5 \max }^{l}, \theta_{5 \min }^{l}\right]$,

- if $\theta_{2 \min } \leq \theta_{2 \text { align }}^{l} \leq \theta_{2 \max }$, then $\left[\theta_{2 \min }, \theta_{2 \max }\right]$ is mapped to $\left[\min \left(\theta_{5 \min }^{l}, \theta_{5 \max }^{l}\right), \theta_{5 \max }\right]$,

- if $\theta_{2 \max }, \theta_{2 \min }<\theta_{2 \mathrm{align}}^{l}$, then $\left[\theta_{2 \min }, \theta_{2 \max }\right]$ is mapped to $\left[\theta_{5 \min }^{l}, \theta_{5 \max }^{l}\right]$;

b) for the small configurations, the following three cases hold:

- if $\theta_{2 \max }, \theta_{2 \min }>\theta_{2 \text { align }}^{s}$, then $\left[\theta_{2 \min }, \theta_{2 \max }\right]$ is mapped to $\left[\theta_{5 \min }^{s}, \theta_{5 \max }^{s}\right]$,

- if $\theta_{2 \min } \leq \theta_{2 \text { align }}^{s} \leq \theta_{2 \max }$, then $\left[\theta_{2 \min }, \theta_{2 \max }\right]$ is mapped to $\left[\theta_{5 \min }, \max \left(\theta_{5 \min }^{\mathrm{s}}, \theta_{5 \max }^{\mathrm{s}}\right)\right]$,

- if $\theta_{2 \max }, \theta_{2 \min }<\theta_{2 \text { align }}^{s}$, then $\left[\theta_{2 \min }, \theta_{2 \max }\right]$ is mapped to $\left[\theta_{5 \max }^{s}, \theta_{5 \min }^{s}\right]$. 


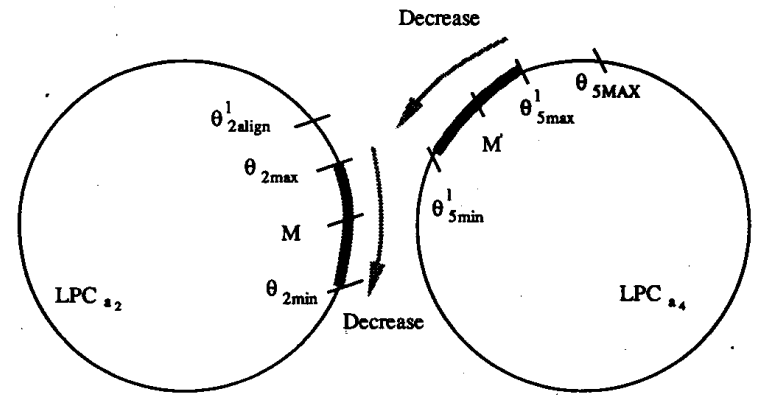

(a)

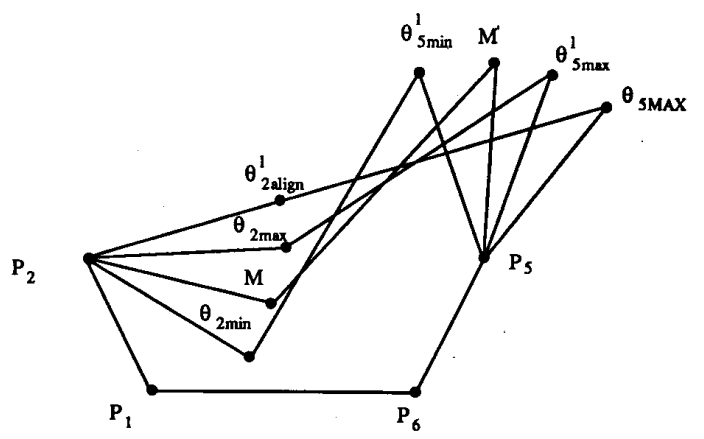

(b)

Fig. 9. If all values in the range $\left[\theta_{2 \min } \theta_{2 \max }\right]$ are smaller than $\theta_{2 \text { align }}^{1}$ then $\left[\theta_{2 \min } \theta_{2 \max }\right]$ is mapped to $\left[\theta_{5 \min }^{l} \theta_{5 \max }^{l}\right]$.

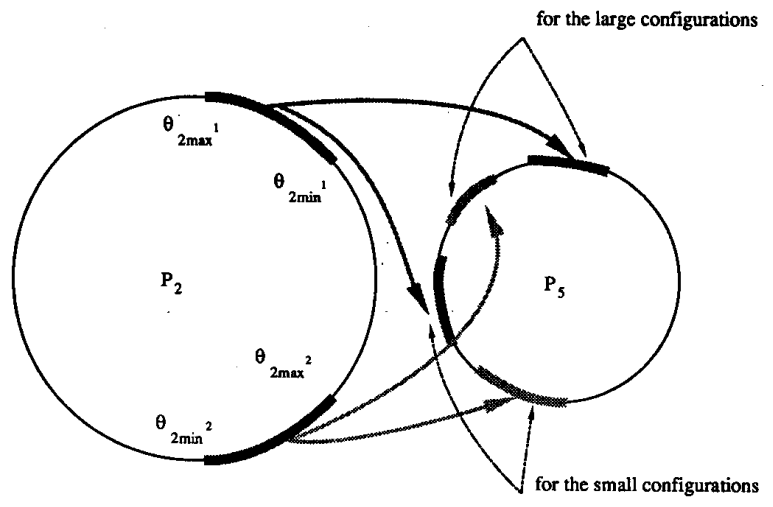

Fig. 10. Two ranges of $\theta_{2}$ can be mapped to four ranges of $\theta_{5}$.

The previous discussion is based on the assumption that the valid region of $R_{\theta_{4}}\left(\theta_{2}\right)$ contain a single range. However, it may consis of two disjoint ranges. If valid region of $R_{\theta_{4}}\left(\theta_{2}\right)$ consists of two disjoint ranges, say, $\left[\theta_{2 \min ^{1}}, \theta_{2 \max ^{1}}\right]$ and $\left[\theta_{2 \min ^{2}}, \theta_{2 \max ^{2}}\right], R_{\theta_{4}}\left(\theta_{5}\right)$ will consist of four ranges (see Fig. 10). They are the ranges for small configurations and large configurations that are mapped from $\left[\theta_{2 \min ^{1}}, \theta_{2 \max ^{1}}\right]$ and the ranges for small configurations and large configurations that are mapped from $\left[\theta_{2 \min ^{2}}, \theta_{2 \max ^{2}}\right]$. The boundaries of these four ranges are determined by applying Algorithm 1.

Decomposing $R_{\theta_{4}}\left(\theta_{5}\right)$ : As described previously, each value of $\theta_{5} \in M R\left(\theta_{5}\right)$ corresponds to two configurations (the small configuration and the large configuration). After mapping $R_{\theta_{4}}\left(\theta_{2}\right)$ to $R_{\theta_{4}}\left(\theta_{5}\right)$, it is guaranteed that for each $\theta_{5} \in R_{\theta_{4}}\left(\theta_{5}\right)$, at least one corresponding configuration has $\theta_{2} \in R_{\theta_{4}}\left(\theta_{2}\right)$ so that at least one corresponding configuration has $\theta_{4} \in\left[90^{\circ}, 270^{\circ}\right]$. However, there is no guarantee that the other configuration has $\theta_{4} \in\left[90^{\circ}, 270^{\circ}\right]$. Since a set of subregions of $M R\left(\theta_{5}\right)$ in which all the configurations satisfy the link collision-free constraint for adjacent links is needed, $R_{\theta_{4}}\left(\theta_{5}\right)$ should be decomposed in order to recognize in which subregions of $R_{\theta_{4}}\left(\theta_{5}\right)$, each $\theta_{5}$ corresponds to two corresponding configurations with $\theta_{2} \in R_{\theta_{4}}\left(\theta_{2}\right)$ and in which subregions of $R_{\theta_{4}}\left(\theta_{5}\right)$, each $\theta_{5}$ corresponds to one small (or large) corresponding configuration with $\theta_{2} \in R_{\theta_{4}}\left(\theta_{2}\right)$. In order to perform this decomposition, the following definition is first be introduced.

Definition 4: Let $M\left(\theta_{5}\right)$ denote any subregion of $M R\left(\theta_{5}\right)$ so that each $\theta_{5} \in M\left(\theta_{5}\right)$ corresponds to two configurations (the large configuration and the small configuration). If in some subregions of $M\left(\theta_{5}\right)$, only the small configurations are being considered, then these subregions are called small-one-configuration regions and are denoted as $S U B_{s}-M\left(\theta_{5}\right)$. If in some subregions of $M\left(\theta_{5}\right)$, only the large configurations are being considered, then these subregions of $M\left(\theta_{5}\right)$ are called large-one-configuration regions and are denoted as $S U B_{l}-M\left(\theta_{5}\right)$. Similarly, if both the large configurations and the small configurations are being considered, they are called twoconfiguration regions and are denoted as $S U B_{2}-M\left(\theta_{5}\right)$.

By Definition $4, R_{\theta_{4}}\left(\theta_{5}\right)$ can be decomposed so that $R_{\theta_{4}}\left(\theta_{5}\right)=$ $S U B_{2}-R_{\theta_{4}}\left(\theta_{5}\right) \cup S U B_{s}-R_{\theta_{4}}\left(\theta_{5}\right) \cup S U B_{1}-R_{\theta_{4}}\left(\theta_{5}\right)$, where $S U B_{2}-R_{\theta_{4}}\left(\theta_{5}\right)$ contains all the subregions of $R_{\theta_{4}}\left(\theta_{5}\right)$ in which each $\theta_{5}$ corresponds to two configurations with $\theta_{2} \in R_{\theta_{4}}\left(\theta_{2}\right)$ and $S U B_{s}-R_{\theta_{4}}\left(\theta_{5}\right)$ ( or $S U B_{l}-R_{\theta_{4}}\left(\theta_{5}\right)$ ) contains all the subregions of $R_{\theta_{4}}\left(\theta_{5}\right)$ in which each $\theta_{5}$ corresponds to one small (or one large ) configuration that has $\theta_{2} \in R_{\theta_{4}}\left(\theta_{2}\right)$.

It can be shown [25] that all the possible subregions of $R_{\theta_{4}}\left(\theta_{5}\right)$ in which each $\theta_{5}$ corresponds to two configurations with $\theta_{2} \in R_{\theta_{4}}\left(\theta_{2}\right)$ will belong to one of the following cases:

1) For each valid range of $R_{\theta_{4}}\left(\theta_{2}\right)$, if the case shown in Fig. 6 occurs then the subregion of $R_{\theta_{4}}\left(\theta_{5}\right)$ that is traveled by $\theta_{5}$ twice when $\theta_{2}$ moves from one boundary to another belongs to $S U B_{2}-R_{\theta_{4}}\left(\theta_{5}\right)$. Thus the region $\left[\theta_{5 \min }^{l}, A\right]$ shown in Fig. 6 , where $A=\theta_{5 \max }$, belongs to $S U B_{2}-R_{\theta_{4}}\left(\theta_{5}\right)$.

2) Recall that the two disjoint subregions of valid $R_{\theta_{4}}\left(\theta_{2}\right)$ can be mapped to four continuous regions of $\theta_{5}$ (see Fig. 10). If $\theta_{5}$ is in the intersection between any two of these four regions of $\theta_{5}$, it can correspond to two configurations with $\theta_{2} \in R_{\theta_{4}}\left(\theta_{2}\right)$.

After finding $S U B_{2}-R_{\theta_{4}}\left(\theta_{5}\right)\left(\theta_{5}\right)$, the subregions for the large and small configurations, $S U B_{l}-R_{\theta_{4}}\left(\theta_{5}\right)$ and $S U B_{s}-R_{\theta_{4}}\left(\theta_{5}\right)$, will be found by identifying the position of $\theta_{2 \mathrm{align}}^{s}$ and $\theta_{2 \mathrm{align}}^{l}$ in $R_{\theta_{4}}\left(\theta_{2}\right)$. As mentioned earlier, each valid range of $R_{\theta_{4}}\left(\theta_{2}\right)$ can be mapped to two ranges of $\theta_{5}$. One is for the small configurations with respect to $\theta_{2}$ and the other is for the large configurations with respect to $\theta_{2}$. It can be shown [25] that in each subregion of $R_{\theta_{4}}\left(\theta_{5}\right)$ which is mapped for the large configurations (or small configurations) with respect to $\theta_{2}$ and is not in $S U B_{2}-R_{\theta_{4}}\left(\theta_{5}\right)$,

1) if a range in this subregion of $\theta_{5}$ is mapped by the range of $\theta_{2}$ in which each $\theta_{2}$ is greater than $\theta_{2 \text { align }}^{l}$ (or $\theta_{2 \text { align }}^{s}$ ), it belongs to $S U B_{l}-R_{\theta_{4}}\left(\theta_{5}\right)$;

2) if a range in this subregion of $\theta_{5}$ is mapped by the range of $\theta_{2}$ in which each $\theta_{2}$ is less than $\theta_{2 \text { align }}^{l}$ (or $\theta_{2 \text { align }}^{s}$ ), it belongs to $S U B_{s}-R_{\theta_{4}}\left(\theta_{5}\right)$.

After finding $S U B_{2}-R_{\theta_{4}}\left(\theta_{5}\right), S U B_{1}-R_{\theta_{4}}\left(\theta_{5}\right)$ and $S U B_{s}$ - $R_{\theta_{4}}\left(\theta_{5}\right)$, if $\theta_{5}$ belongs to one of them, all the corresponding configurations have $\theta_{4} \in\left[90^{\circ}, 270^{\circ}\right]$. As mentioned earlier, for each $\theta_{5} \in R_{\theta_{2}, \theta_{3}}\left(\theta_{5}\right)$, all the corresponding configurations have $\theta_{2}$ in its valid range $\left[0^{\circ}, 360^{\circ}\right]$ and $\theta_{3}$ in its valid range $\left[90^{\circ}, 270^{\circ}\right]$. 
Consequently, if $\theta_{5} \in M R\left(\theta_{5}\right) \cap R_{\theta_{2}, \theta_{3}}\left(\theta_{5}\right) \cap R_{\theta_{4}}\left(\theta_{5}\right)$, the corresponding configurations will satisfy the link collision-free constraint for adjacent links. However, for doing these intersection, attention should be paid to the facts that $M R\left(\theta_{5}\right)$ and $R_{\theta_{2}, \theta_{3}}\left(\theta_{5}\right)$ are two-configuration regions; and $R_{\theta_{4}}\left(\theta_{5}\right)$ is the union of twoconfiguration regions, $S U B_{s}-M\left(\theta_{5}\right) \mathrm{s}$ and $S U B_{l}-M\left(\theta_{5}\right) \mathrm{s}$.

Finding Subregions of $M R\left(\theta_{5}\right)$, which Satisfy the Link CollisionFree Constraint for Nonadjacent Links: To satisfy the link collisionfree constraint for nonadjacent links, it can be observed from a 6-link closed chain that:

1) Since $B C U\left(a_{2}, a_{3}, a_{4}\right)$ is being discussed, the positions of links $a_{1}, a_{5}$ and $a_{6}$ are fixed. The easily identified values of $\theta_{1}$ and $\theta_{6}$ that result in a collision of these links need not be further analyzed.

2) The collision between the two nonadjacent changeable links $a_{2}$ and $a_{4}$ can be avoided by imposing appropriate limits to $\theta_{3}$ and $\theta_{4}$. Here it is assumed that $\theta_{3}$ and $\theta_{4}$ are within the range $\left[90^{\circ}, 270^{\circ}\right]$.

3) The changeable links $a_{2}, a_{3}$ and $a_{4}$ can possibly intersect the fixed links $a_{1}, a_{5}$ or $a_{6}$. Since $\theta_{3}$ and $\theta_{4}$ must be in the range $\left[90^{\circ}, 270^{\circ}\right]$, the changeable links $a_{2}, a_{3}$ or $a_{4}$ can possibly intersect the fixed links $a_{1}, a_{5}$ or $a_{6}$ only if $\theta_{5}$ or $\theta_{2}$ becomes small.

The values of $\theta_{5 \text { contact }}$ (or $\theta_{2 \text { contact }}$ ), defined as the value of $\theta_{5}$ (or $\theta_{2}$ ) when one of $a_{3}$ and $a_{4}$ (or one of $a_{3}$ and $a_{2}$ ) touches $a_{1}, a_{5}$ or $a_{6}$, can be found according to the geometric relationships of the 6-link closed chain. Subsequently, a subregion $R_{n o n}\left(\theta_{5}\right)$ of $M R\left(\theta_{5}\right)$ in which each configuration has $\theta_{5} \geq \theta_{5 \text { contact }}$ and $\theta_{2} \geq \theta_{2 \text { contact }}$ can be obtained by the method described in the previous sections. Hence, if $\theta_{5} \in R_{n o n}\left(\theta_{5}\right)$, the link collision-free constraint for nonadjacent links can be satisfied. Consequently, we can conclude that the feasible region $F R\left(\theta_{5}\right)$ in which the link collision-free constraints are satisfied is the intersection between the ranges that satisfy the link-collision-free constraints for adjacent links and the ranges that satisfy the link-collision-free constraint for nonadjacent links. Thus all of the feasible configurations are included in this feasible region.

\section{Finding All the Collision-Free Feasible Configurations}

In the last section it was shown how the feasible regions $F R\left(\theta_{5}\right)$ could be computed. If there are obstacles in the environment, then any collision with these obstacles should also be avoided. In this section, the subregions of $F R\left(\theta_{5}\right)$ in which each feasible configuration is collision-free will be found. In the following discussion, a singleobstacle environment is studied. For an environment with multiple obstacles, the principle of superposition can be applied so that the subregions of $F R\left(\theta_{5}\right)$ in which each feasible configuration is collision-free are equal to the intersection of those in each singleobstacle environment. The simplest case in which the link widths, i.e., $w_{O}$ and $w_{R}$, are equal to zero and the obstacle is a convex polygon is presented. If the obstacle is a concave polygon, it can be decomposed as a combination of several convex polygons. If $w_{O}$ and $w_{R}$ are not equal to zero, the results can be modified by shrinking the width of the carried object and the robot arms to zero and enlarging the size of the obstacle accordingly [2], [3]. Generally speaking, this method becomes cumbersome when arbitrary orientations are allowed. However, in this case, one is dealing with a constraint mechanism so that general orientations are not allowed. In the following discussion, it will be shown that the algorithm is only concerned with the distance between the corner or edge of the obstacle and the outer boundary of the carried object (link $a_{3}$ ). This allows the obstacles to be grown in a straight forward manner.

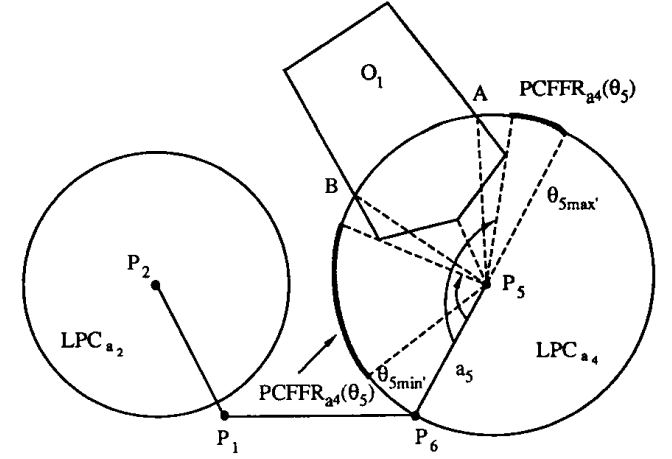

Fig. 11. An illustration for finding $\operatorname{PCFFER}_{a^{4}}\left(\theta_{5}\right)$.

Now, assuming there is one convex obstacle in the workspace and the widths of the carried object and robot links are zero (i.e., $w_{O}=0$ and $w_{R}=0$ ), subregions of $F R\left(\theta_{5}\right)$, which satisfy the collision-free constraint can be obtained by considering the collisionfreeness of each changeable link of $a_{2}, a_{3}$ and $a_{4}$ one by one in $B C U\left(a_{2}, a_{3}, a_{4}\right)$. The procedure is given in the following:

1) Find subregions of $F R\left(\theta_{5}\right)$ in which the corresponding positions of $a_{4}$ do not have any collision with the obstacle. Such a subregion is called a possible collision-free feasible region of $a_{4}$ and is denoted PCFFR $R_{a_{4}}\left(\theta_{5}\right)$.

2) Find subregions of $P C F F R_{a_{4}}\left(\theta_{5}\right)$ in which the corresponding positions of $a_{4}$ and $a_{2}$ do not have any collision with the obstacle. Similarly, each of these subregions is called a possible collision-free feasible region of $a_{2}$ and $a_{4}$ and is denoted PCFFR $R_{a_{2}, a_{4}}\left(\theta_{5}\right)$.

3) Find subregions of $P C F F R_{a_{2}, a_{4}}\left(\theta_{5}\right)$ in which the corresponding positions of all changeable links $a_{2}, a_{3}$ and $a_{1}$ are collision-free with the obstacle. Each of these subregions is called a collision-free feasible region and is denoted $C F F R\left(\theta_{5}\right)$ or simply a CFFR.

Each step is discussed in detail in the following subsections.

Finding PCFF $R_{a_{4}}\left(\theta_{5}\right)$ and $P C F F R_{a_{2}, a_{4}}\left(\theta_{5}\right)$ : Suppose there is an obstacle $O_{1}$ in the environment that intersects $L P C_{a_{4}}$ at points $A$ and $B$, where $\theta_{5 A}>\theta_{5 B}$ (see Fig. 11). To find $P C F F R_{a_{4}}\left(\theta_{5}\right)$, a set of rays from $P_{5}$ to $\mathrm{A}, \mathrm{B}$ and to each corner of $O_{1}$ which is inside $L P C_{a_{4}}$ can be obtained. The angles from link $a_{5}$ to each ray are calculated, then the smallest and largest angles $\theta_{5 B^{\prime}}$ and $\theta_{5 A^{\prime}}$ among them are selected. Assuming $\theta_{\max }^{\prime}$ and $\theta_{\min }{ }^{\prime}$ are the largest and smallest angles of $F R\left(\theta_{5}\right)$, then $\operatorname{PCFFR}_{a_{4}}\left(\theta_{5}\right)=$ $\left[\theta_{5 A^{\prime}}, \theta_{5 \text { max }^{\prime}}\right]+\left[\theta_{5 \min ^{\prime}}, \theta_{5 B^{\prime}}\right]$. By a similar approach, the subregions of $a_{2}$ in which $a_{2}$ does not collide with an obstacle can be obtained. Subsequently, by using the method discussed in the previous sections, these subregions of $\theta_{2}$ are mapped to the ranges of $\theta_{5}$, which

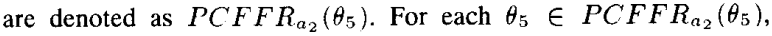
in the corresponding configurations, there is no collision between

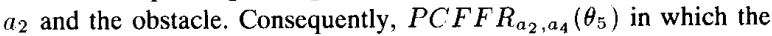
corresponding positions of $a_{4}$ and $a_{2}$ do not have any collision with the obstacle is equal to $P C F F R_{a_{4}}\left(\theta_{5}\right) \cap P C F F R_{a_{2}}\left(\theta_{5}\right)$.

Finding CFFR: Now our goal is to find collision-free feasible regions (CFFR's) that are subregions of $P C F F R_{a_{2}, a_{4}}\left(\theta_{5}\right)$ and in which all the changeable links $a_{2}, a_{3}$ and $a_{4}$ are collision-free with the obstacle. For convenience, $P C F F R_{a_{2}, a_{4}}\left(\theta_{5}\right)^{i}$ and $C F F R^{i}$, where $\mathrm{i}=2, s, l$, are used to represent a $P C F F R_{a_{2}, a_{4}}\left(\theta_{5}\right)$ and a CFFR, which is a two-configuration region, a $S U B_{s}-M\left(\theta_{5}\right)$, or a $S U B_{l}-M\left(\theta_{5}\right)$. respectively. Since a $C F F R^{i}$ is a subregion of a $P C F F R_{a_{2}, a_{4}}\left(\theta_{5}\right)^{i}$, there should be some collision-free feasible con- 


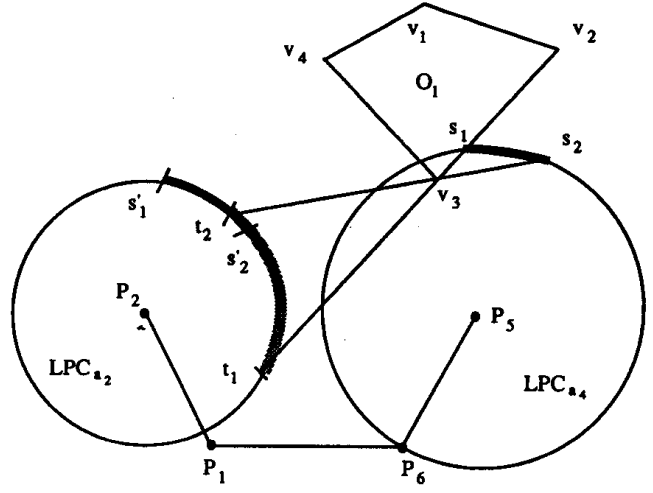

Fig. 12. An illustration of the technique used for finding critical CFFC's that determines the boundaries of collision-free feasible regions.

figurations (CFFC's) that divide $P C F F R_{a_{2}, a_{4}}^{i}$ into several nonzero subregions. This kind of CFFC in a $P C F F R_{a_{2}, a_{4}}\left(\theta_{5}\right)^{i}$ is called a critical CFFC, since the subregion that is at one side of the CFFC is a CFFR, but the one that is at the other side of the CFFC is not collisionfree. Obviously, if all the critical CFFC's can be obtained, then all the CFFR's can be found as well. Subsequently, all the CFFC's can be found. To find the critical CFFC's, the following lemma is introduced.

Lemma 1: If a 6-link closed chain is in a critical CFFC of a $P C F F R_{a_{2}, a_{4}}\left(\theta_{5}\right)^{i}$, then $a_{3}$ must pass through a corner or an edge of the obstacle.

It is easy to show that this lemma is true since link $a_{3}$ is a line segment and the obstacle is a convex polygon.

By Lemma 1, the critical CFFC's can be found by finding the CFFC's in which the location of $a_{3}$ touches a corner or an edge of the obstacle. Now let's consider a critical CFFC that touches a corner of an obstacle. In this critical CFFC, $a_{3}$ should touch a corner of the obstacle; $\theta_{5}$ should be in a PCFF $R_{a_{2}, a_{3}}\left(\theta_{5}\right)$; and $\theta_{2}$ should be in $P C F F R_{a_{2}}\left(\theta_{2}\right)$, which is the mapping of $P C F F R_{a_{2}, a_{3}}\left(\theta_{5}\right)$. Our strategy to find this critical CFFC is described as follows: 1) for each $P C F F R_{a_{2}, a_{3}}\left(\theta_{5}\right)$, find its mapping on $\theta_{2}$, i.e., $P C F F R_{a_{2}}\left(\theta_{2}\right)$; 2) since not all the configurations on $P C F F R_{a_{2}}\left(\theta_{2}\right)$ can touch the corner of the obstacle, find the subrange of $P C F F R_{a_{2}}\left(\theta_{2}\right)$ in which the configurations can possibly touch the corner of the obstacle; (3) find the subrange of $P C F F R_{a_{2}, a_{3}}\left(\theta_{5}\right)$ in which the configurations possibly touch the corner of the obstacle and have one end of $a_{3}$ on the range obtained in Step 2; (4) use the geometric relationships to locate a configuration in which $a_{3}$ passes through a corner of the obstacle and has its two ends at the ranges obtained in Step 2 and Step 3 respectively.

Before giving the details of the algorithm, for convenience, a notation that builds the relationship between $\theta_{5}\left(\theta_{2}\right)$ and a point on the circle $L P C_{a_{4}}\left(L P C_{a_{2}}\right)$ is first introduced. In the following discussion, as each position of $a_{4}$ (or $a_{2}$ ) corresponds to a distinct value of $\theta_{5}$ (or $\theta_{2}$ ), it is assumed that if the changeable end of the centerline of $a_{4}$ (or $\left.a_{2}\right)$ is at a point $\mathrm{P}$ on $L P C_{a_{4}}$ (or $L P C_{a_{2}}$ ), the corresponding $\theta_{5}$ (or $\theta_{2}$ ) is denoted as $\theta_{5 P}$ (or $\theta_{2 P}$ ). Suppose one is given a $\left[\theta_{5 s_{1}}, \theta_{5 s_{2}}\right]$ which is a $P C F F R_{a_{2}, a_{4}}\left(\theta_{5}\right)^{i}(\mathrm{i}=\mathrm{s}, l)$ on $L P C_{a_{4}}$, and $v_{3}$ which is a corner of the obstacle (see Fig. 12), an algorithm that locates the critical CFFC within $P C F F R_{a_{2}, a_{4}}\left(\theta_{5}\right)^{i}$ and touching corner $v_{3}$ is given as following.

Algorithm 2:

1) Find $P C F F R_{a_{2}}\left(\theta_{2}\right)=\left[\theta_{2 s_{1}^{\prime}}, \theta_{2 s_{2}^{\prime}}\right]$ which is the mapping of $P C F F R_{a_{2}, a_{4}}\left(\theta_{5}\right)^{i}$ on $L P C_{a_{2}}$.

2) Find the subrange $\left[T_{1}, T_{2}\right]$ of $P C F F R_{a_{2}}\left(\theta_{2}\right)=\left[\theta_{2 s_{1}^{\prime}}, \theta_{2 s_{2}^{\prime}}\right]$

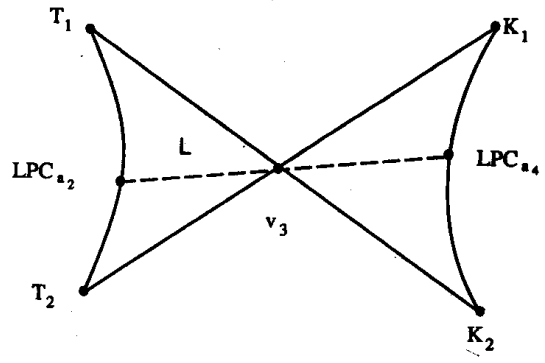

Fig. 13. The region in which a critical CFFC may exist.

in which the configurations can possibly touch the corner of the obstacle by calculating the possible intersection of line $s_{1} v_{3}$ with $L P C_{a_{2}}\left(P_{2}\right)$, denoted $t_{1}$ and the intersection of line $v_{3} s_{2}$ with $L P C_{a_{2}}\left(P_{2}\right)$, denoted $t_{2}$.

a) If both intersections do not exist or the intersection of the two arcs $t_{1} t_{2}$ and $s_{1} s_{2}$ is empty, then $\left[T_{1}, T_{2}\right]$ is empty, i.e., there is no critical CFFC with respect to the corner $v_{3}$. Stop.

b) If both $t_{1}$ and $t_{2}$ exist and the intersection between arcs $s_{1}^{\prime} s_{2}^{\prime}$ and $t_{1} t_{2}$ is not empty, $\left[T_{1} T_{2}\right]$ is equal to this intersection, i.e., $t_{1} t_{2} \cap s_{1}^{\prime} s_{2}^{\prime}$.

c) If there exists only one $t_{i}, i=1$ or 2 , the line $t_{i} s_{i}$ would separate the entire plane into two subplanes and separate $L P C_{a_{2}}$ into two arcs.

- If the intersection between $s_{1}^{\prime} s_{2}^{\prime}$ and one arc of $L P C_{a_{2}}$ which is in the same subplane with line $s_{j} v_{3}$, where $i, j=1,2, i \neq j$, is empty, then $\left[T_{1}, T_{2}\right]$ is empty, i.e., there is no critical CFFC with respect to the corner $v_{3}$. Stop.

- Otherwise, $T_{1} T_{2}$ is equal to $s_{1}^{\prime} s_{2}^{\prime}$.

3) Find the subrange $\left[K_{1}, K_{2}\right]$ of $P C F F R_{a_{2}, a_{3}}\left(\theta_{5}\right)$ in which the configurations possibly touch the corner of the obstacle and have one end of $a_{3}$ on the range obtained in Step 2 by calculating the possible intersection of line $T_{1} v_{3}$ with $L P C_{a_{4}}\left(P_{5}\right)$, denoted $K_{1}$ and the intersection of line $T_{2} v_{3}$ with $L P C_{a_{4}}\left(P_{5}\right)$, denoted $K_{2}$ (see Fig. 13).

4) Use a set of equations derived from the geometric properties of the area shown in Fig. 13 [25] to find a line segment $L$ with length $l_{3}$, which has one end on $\left[T_{1}, T_{2}\right]$ of $L P C_{a_{2}}$, one end on $\left[K_{1}, K_{2}\right]$ of $L P C_{a_{4}}$, and passes through $v_{3}$. If line segment $L$ exists, then a feasible position of $a_{3}$ in the area shown in Fig. 13 can be found. This feasible position of $a_{3}$ defines a critical CFFC.

After checking each corner of the obstacle, the edges of the obstacle should also be checked by overlapping link $a_{3}$ with the edges of the obstacle. If there is a collision-free feasible configuration in which link $a_{3}$ and an edge of the obstacle overlap, it is a critical CFFC. After finding all the critical CFFC's in all the $P C F F R_{a_{2}, a_{4}}\left(\theta_{5}\right)$ 's, all the CFFR's and hence all the CFFC's for a single-obstacle environment can be determined.

\section{Finding a Collision-Free Path}

In the previous section, all of the CFFC's in $B C U\left(a_{2}, a_{3}, a_{4}\right)$ in terms of $\theta_{5}$ have been found for a set of quantized values of $\theta_{1}$ and $\theta_{6}$. Since $\theta_{1}, \theta_{6}$ and $\theta_{5}$ have been chosen as the independent variables, the collision-free path finding problem with these three independent variables can be solved in the following four steps:

1) Find the transitions between $C F F R^{i}$ 's $(\mathrm{i}=2, l, s)$ at fixed values of $\theta_{1}$ and $\theta_{6}$ 
2) Find the transitions between $C F F R^{i}$ 's at adjacent ( quantized ) values of $\theta_{1}$ and $\theta_{6}$.

3) Build a connection graph in which each vertex is a $C F F R^{i}$ in $B C U\left(a_{2}, a_{3}, a_{4}\right)$ for a specific $\theta_{1}$ and $\theta_{6}$, and each edge is a transition between a pair of $C F F R^{i}$ 's at fixed values of $\theta_{1}$ and $\theta_{6}$ or at adjacent ( quantized) values of $\theta_{1}$ and $\theta_{6}$.

4) Search a collision-free path for the 6-link closed chain in the connection graph.

The details are discussed in the following subsections.

A. Finding Transitions Between $C F F R^{i}$ 's $(i=2, s, l)$ In different $B C U\left(a_{2}, a_{3}, a_{4}\right)^{\prime} s$

For a specific $\theta_{1}$ and $\theta_{6}$, there is one degree of freedom that is represented by $\theta_{5}$ in $B C U\left(a_{2}, a_{3}, a_{4}\right)$. A transition is defined to exist between two $C F F R^{i}$ s $(\mathrm{i}=2, l, s)$ in $B C U\left(a_{2}, a_{3}, a_{4}\right)$ if when the value of $\theta_{5}$ is changed continuously, a CFFC in one $C F F R^{i}$ can be automatically transferred to a CFFC in another $C F F R^{i}$. There are three possible types of transitions based on the combination of different types of CFFRs: (1) transitions between $C F F C^{i}$ s $(i=2, l, s)$ in separate $C F F R^{i}$ s, (2) transitions between CFFC's in a $C F F R^{i}(i \neq 2)$ and a $C F F R^{2}$, and (3) transitions between a $C F F C^{l}$ and a $C F F C^{s}$ in a $C F F R^{2}$. Since there is only one degree of freedom in the $B C U\left(a_{2}, a_{3}, a_{4}\right)$, by definition the transitions between two separate CFFR's cannot exist. Therefore, the transitions in the case 1 discussed previously cannot exist, and only special situations in the case 2 , and 3 can exist. These special situations are listed as follows:

1) a $C F F R^{l}$ and a $C F F R^{l}$ which belongs to a $C F F R^{2}$ (or a $C F F R^{s}$ and a $C F F R^{s}$ which belongs to a $C F F R^{2}$ ) contain a common $\theta_{5}$, i.e, they are connected by a point on $L P C_{a_{4}}$.

2) If in a two-configuration region $C F F R^{2}$, there is a point at which $a_{2}$ and $a_{3}$ become aligned, then the $C F F R^{s}$ and the $C F F R^{l}$ which belong to the $C F F R^{2}$ can be transferred to each other. The point at which $a_{2}$ and $a_{3}$ become aligned is normally at the boundary of the motion region $M R\left(\theta_{5}\right)$.

B. Finding Transitions between $C F F R^{i}$ 's $(i=2, s, l)$ in Different $B C U\left(a_{2}, a_{3}, a_{4}\right)^{\prime} s$

In addition to the transitions between $C F F R^{i}$ 's $(i=2, s, l)$ in a $B C U\left(a_{2}, a_{3}, a_{4}\right)$ with the same $\theta_{1}$ and $\theta_{6}$, transitions between $C F F R^{i}$ 's in two different $B C C\left(a_{2}, a_{3}, a_{4}\right)^{\prime} s$ which have different quantized values of $\theta_{1}$ or different quantized values of $\theta_{6}$ need to be found. For each BCU, there are three kinds of CFFR's: $C F F R^{2}$ (which is composed of a $C F F R^{l}$ and $C F F R^{s}$ ), $C F F R^{l}$ and $C F F R^{s}$. In the following discussion, for convenience, it is assumed that (1) a $C F F R^{2}$ is considered as an independent $C F F R^{l}$ and an independent $C F F R^{s}$, (2) if a $C F F R^{j}(j=l, s)$ and a $C F F R^{j}$ which belongs to a $C F F R^{2}$ are connected by a point, they are considered as one $C F F R^{j}$. Due to this assumption, for adjacent BCU's, only transition between the same kind of $C F F R^{\prime} s$, i.e. between $C F F R^{l}$ 's or $C F F R^{s}$ 's and transition between $C F F R^{l}$ and $C F F R^{s}$ need to be discussed. In the following, transitions between CFFR's in two adjacent BCU's, which have have different (quantized) values of $\theta_{1}$ and the same value of $\theta_{6}$ are discussed. Transitions for BCU's with the same value of $\theta_{1}$ but adjacent values of $\theta_{6}$ can be obtained in a similar manner.

For convenience, the notation $\left.B C C\left(a_{2}, a_{3}, a_{4}\right)\right|_{\theta_{1}^{m}, \theta_{6}^{n}}$ will be used to represent $B C U\left(a_{2}, a_{3}, a_{4}\right)$ at a specific value of $\theta_{1}$ $\left(\theta_{1}^{m}\right.$ in this case) and a specific value of $\theta_{6}\left(\theta_{6}^{n}\right.$ in this case), and $C F F R^{i}(k)_{\theta_{1}^{m}, \theta_{6}^{n}}$ is used to represent the k-th $C F F R^{i}(\mathrm{i}=\mathrm{s}$ or $l)$ in $\left.B C U\left(a_{2}, a_{3}, a_{4}\right)\right|_{\theta_{1}^{m}, \theta_{6}^{n}}$. First let's discuss the transitions between the same type of the CFFR's.
Assume there are two of the same type of collision-free feasible regions: $C F F R^{i}(k)_{\theta_{1}^{m}, \theta_{6}^{n}}$ in $\left.B C U\left(a_{2}, a_{3}, a_{4}\right)\right|_{\theta_{1}^{m}, \theta_{6}^{n}}$ and

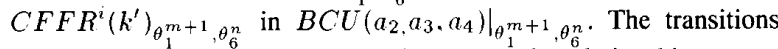
between them will be based on the geometric relationship among two corresponding BCU's. Since these two CFFR's have different values of $\theta_{1}$ but the same value of $\theta_{6}$, the corresponding BCU's, i.e., $\left.B C U\left(a_{2}, a_{3}, a_{4}\right)\right|_{\theta_{1}^{m}, \theta_{6}^{n}}$ and $\left.B C U\left(a_{2}, a_{3}, a_{4}\right)\right|_{\theta_{1}^{m+1}, \theta_{6}^{n}}$ also have the same value of $\theta_{6}$. Therefore, they have the same link position circles $L P C_{a_{4}}$. As mentioned earlier, for each $\mathrm{BCU}, C F F R^{i}$ 's are separated by obstacles and therefore one $C F F R^{i}$ cannot be transferred to another $C F F R^{i}$ in a specific BCU. Thus if there is a transition between $C F F R^{i}(k)_{\theta_{1}^{m}, \theta_{6}^{n}}$ and $C F F R^{i}\left(k^{\prime}\right)_{\theta_{1}^{m+1}, \theta_{6}^{n}}$, $C F F R^{i}(k)_{\theta_{1}^{m}, \theta_{6}^{n}} \cap C F F R^{i}\left(k^{\prime}\right)_{\theta_{1}^{m+1}, \theta_{6}^{n}}$ must not be empty. If the quantization intervals of $\theta_{1}{ }^{1}$ and ${ }^{6} \theta_{6}$ are sufficiently small then in the case $C F F R^{i}(k)_{\theta_{1}^{m}, \theta_{6}^{n}}$ and $C F F R^{i}\left(k^{\prime}\right)_{\theta_{1}^{m+1}, \theta_{6}^{n}}$ are collision-free at quantized values $\theta_{1}^{m}$ and $\theta_{1}^{m+1}$ for both BCU,

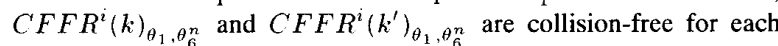
value of $\theta_{1}$ in the interval between $\theta_{1}^{m}$ and $\theta_{1}^{m+1}$. Consequently, if $\operatorname{CFFR}^{i}(k)_{\theta_{1}^{m}, \theta_{6}^{n}} \cap C F F R^{i}\left(k^{\prime}\right)_{\theta_{1}^{m+1}, \theta_{6}^{n}} \neq 0$, then there is a transition between $C F F R^{i}(k)_{\theta_{1}^{m}, \theta_{6}^{n}}$ and $C F F R^{i}\left(k^{\prime}\right)_{\theta_{1}^{m+1}, \theta_{6}^{n}}$. If there are two different types of collision-free feasible regions $C F F R^{i}(k)_{\theta_{1}^{m}, \theta_{0}^{n}}$ and $C F F R^{j}\left(k^{\prime}\right)_{\theta_{1}^{m+1}, \theta_{6}^{n}}$ where $\mathrm{i}, \mathrm{j}=\mathrm{s}, l, i \neq j$, whether there is a transition between them depends on if there is a point in $C F F R^{i}(k)_{\theta_{1}^{m}, \theta_{6}^{n}} \cap C F F R^{j}\left(k^{\prime}\right)_{\theta_{1}^{m+1}, \theta_{6}^{n}}$, which can make $a_{2}$ and $a_{3}$ become aligned, because it is the only case in which the large configuration can be transferred to the small configuration.

After finding all CFFR's and all the possible transitions among them, a connection graph can be built. In this connection graph, each vertex is a CFFR and each edge is a transition among CFFR's. Since there are three degrees of freedoms, the connection graph can be represented in a three-dimensional space with independent variables $\theta_{1}, \theta_{6}$ and $\theta_{5}$. Because the connection graph represents all the free space in the joint space of the 6-link closed chain, a collision-free path of the closed chain can be searched in this connection graph.

\section{An Example}

The following example illustrates the construction of a connection graph. After all of the CFFR 's for the 6-link closed chain have been found in term of $\theta_{1}, \theta_{5}$ and $\theta_{6}$, a connection graph can be described in a 3-D space for different values of $\theta_{1}, \theta_{5}$ and $\theta_{6}$, where $\theta_{1}$ and $\theta_{6}$ are chosen as the axes in the horizontal plane and $\theta_{5}$ is chosen as the vertical axis. In this example, variables $\theta_{1}$ and $\theta_{6}$ are quantized. A point on the $\theta_{1}-\theta_{6}$ plane which corresponds to a specific quantized value of $\theta_{1}$ and $\theta_{6}$ defines a unique $\left.B C C^{-}\left(a_{2}, a_{3}, a_{4}\right)\right|_{\theta_{1}, \theta_{6}}$. Since there is only one degree of freedom of motion in $\left.B C U\left(a_{2}, a_{3}, a_{4}\right)\right|_{\theta_{1}, \theta_{6}}$, vertical line segments which originate from a point on $\theta_{1}-\theta_{6}$ plane and parallel to the $\theta_{5}$ axis can represent all the $C F F R^{i}$,s in $\left.B C U\left(a_{2}, a_{3}, a_{4}\right)\right|_{\theta_{1}, \theta_{6}}$ (with two parallel segments to denote two-configurations). These CFFR's are the vertices of the connection graph (see Fig. 14). If there is a transition between two of these $C F F R^{i}$ 's, an edge in the connection graph can be drawn (shown as a dashed line in Fig. 14). After all the transitions between $C F F R^{i}$ 's $(i=2, s, l)$ are found, a connection graph is built. Consequently, a collision-free path for the 6-link closed chain can be obtained by applying a graph search method in the connection graph.

\section{CONCLUSion}

This paper has discussed an off-line approach for planning a collision-free path for two robot manipulators that cooperate in 


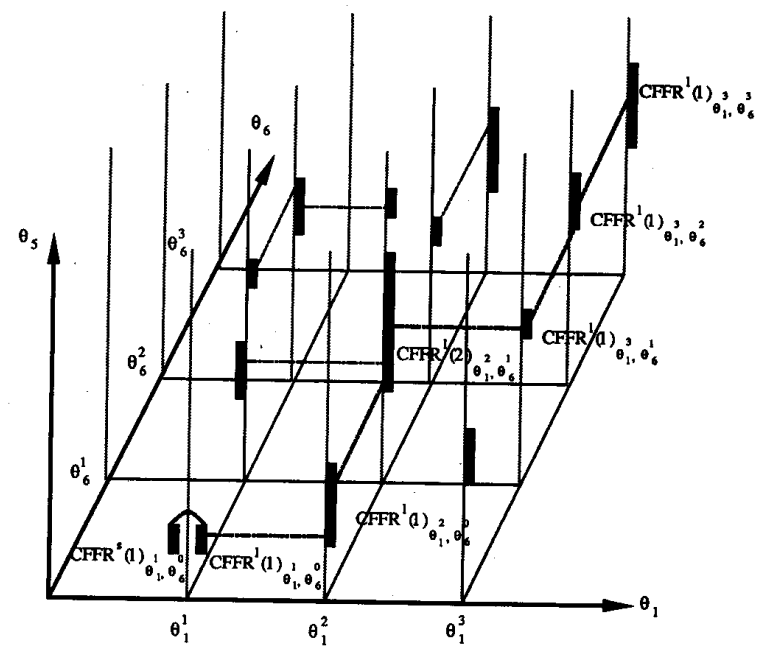

Fig. 14. A connection graph showing all possible collision-free feasible configurations for a 6-link closed chain. A collision-free path of the 6-link closed chain from initial position $\operatorname{CFFR}^{s}(1)_{\theta_{1}^{3} \theta_{6}^{3}}$ to final position $\operatorname{CFFR}^{1}(1)_{\theta_{1}^{3} \theta_{6}^{3}}$, which is shown by bold dashed lines.

carrying a rectangular object in a 2-D environment. The central concept of this approach is to map the free space in the Cartesian world space to the robot's joint space. In the joint free space, each joint configuration of the robots satisfies the closed-chain constraint, the link collision-free constraints, and the obstacle collision-free constraint. A collision-free path for the robots is then computed in this joint free space. Two major algorithms, namely the collisionfree feasible configuration finding algorithm and the collision-free path finding algorithm, are employed in this approach. Although the approach described here is precise enough to deal with a cluttered environment, it may still be regarded as an approximation method in practice. This is because (1) $\theta_{1}$ and $\theta_{6}$ are quantized, and (2) the carried object is approximated by a rectangle. To relax the second assumption, the results reported here are currently being expanded to polygonal objects.

\section{REFERENCES}

[1] Y. F. Zheng and F. R. Sias, Jr., "Two robot arms in assembly," in Proc. IEEE Int. Conf. Robotics Automat., 1986, pp. 1230-1235.

[2] T. Lozano-Perez, "Automatic Planning of Manipulator Transfer Movements," IEEE Trans. Syst., Man, Cybern., vol. SMC-11, pp. 681-698, Oct. 1981.

[3] T. Lozano-Perez, "Spatial planning: a configuration space approach," IEEE Trans. Comput., vol. C-32, pp. 26-38, February 1983.

[4] J. T. Schwartz and M. Sharir, "On the piano movers' problem: Part I-The special case of a rigid polygonal body moving amidst polygonal barries," Commun. Pure Appl. Math., vol. 36, pp. 345-398, 1983.

[5] J. T. Schwartz and M. Sharir, "On the piano movers' Problem: Part II-General techniques for computing topological properties of real algebraic manifolds," Adv. Appl. Math., pp. 298-351, 1983.

[6] J. T. Schwartz and M. Sharir, "On the Piano Movers' Problem: Part III-Coordinating the motion of several independent bodies: the special case of circular bodies moving amidst polygonal barriers,"The Int. $J$. Robotics Res., vol. 2, no. 3, pp. 46-75, Fall 1983.

[7] L. Gouzenes, "Strategies for solving collision-free trajectories problems for mobile and manipulator robots," The Int. J. Robotics Res., vol. 3, pp. $51-65,1984$.

[8] C. Bajaj and M. Kim, "Generation of configuration space obstacles-Part I: The case of a moving sphere," Tech. Report CSD-TR-565, Dept. Comput. Sci., Purdue Univ., W. Lafayette, IN, Dec. 1985.

[9] M. Erdmann and T. Lozano-Perez, "On multiple moving objects," in Proc. IEEE Int. Conf. Robotics Automat., 1986, pp. 1419-1424.
[10] T. Lozano-Perez, "A simple motion-planning algorithm for general robot manipulators," IEEE J. Robotics Automat, vol. RA-3, pp. 224-238, June 1987.

[11] K. Kedem and M. Sharir, "An efficient motion planning algorithm for a convex polygonal object in two dimensional polygonal space," Tech. Rep. No. 253, Comput. Sci. Dept., Courant Institute, New York, NY, Oct. 1986.

[12] Q. Ge and J. M. McCarthy, "Equations for boundaries of joint obstacles for planar robots," in Proc. 1989 IEEE Int. Conf. Robotics Automat., vol. 1, May 1989, pp. 164-169.

[13] Y. K. Hwang and N. Ahuja, "Path planning using a potential field representation," in Proc. IEEE Int. Conf. Robotics Automat., 1988, pp. 648-649.

[14] Y. F. Zhang and J. Y. S. Luh, "Constrainted relations between two coordinated industrial robots," in Proc. 3rd Annu. Conf. Intelligent Syst. and Machines, Apr. 1985, Rochester, MI, pp. 118-123.

[15] H. Hemami, "A state model for interconnected rigid bodies," IEEE Trans. Automat. Contr., vol. AC-27, Apr. 1982.

[16] S. Chand and K. L. Doty, "Trajectory-specification and load distribution for closed-loop multi-manipulator system," Amer. Contr. Conf., 1985, Boston, MA.

[17] $Y$. Chen and A. A. Desrochers, "A proof of the structure of the minimum-time control law of robot manipulators using a hamiltonian formulation," IEEE Trans. Robotics Automat., vol. 6, pp. 388-393, June 1900.

[18] E. Freund and H. Hoyer, "Hierarchical control of guided collision avoidance for robots in automatic assembly," in Proc. 4th Int. Conf. Assembly Automat., Japan, Sept. 1983, pp. 91-103.

[19] E. Freund and H. Hoyer, "Pathfinding in multi-robot systems: Solution and applications," in Proc. IEEE Int. Conf. Robotics Automat., San Francisco, CA, 1986, pp. 103-111.

[20] S. Fortune, G. Wilfong and C. Yap, "Coordinated motion of two robot arms," in Proc. IEEE Int. Conf. Robotics Automat., 1986, pp. 1216-1223.

[21] R. Zapata, A. Fournier, and P. Dauchez, "True cooperation of robot in multi-arms tasks," in Proc. IEEE Int. Conf. Robotics Automat., 1987, pp. $1255-1260$.

[22] V. J. Lumelsky and A. A. Stepanov, "Dynamic path planning for a mobile automaton with limited information on the environment," IEEE Trans. Automat. Contr., vol. AC-31, Nov. 1986.

[23] H. H. Mabie and C. F. Reinholtz, Mechanisms and Dynamics of Machinery, fourth ed. New York: Wiley, 1987.

[24] A. Midha, Z. L. Zhao, and I. Her, "Mobility conditions for planar linkage using triangle inequality and graphical interpretation," Trans. ASME vol 107 , Sept. 1985.

[25] Q. Xue, "Path planing for mobile robots with manipulators," Ph.D. dissertation, Purdue Univ., W. Lafayette, 1990. 